\title{
Civilisations
}

Revue internationale d'anthropologie et de sciences

humaines

$54 \mid 2006$

Expériences de recherche en République

démocratique du Congo

\section{5 ans de recherches en RDC}

Permanences et renouvellement des méthodes

\section{Léon de Saint Moulin}

\section{(2) OpenEdition}

\section{Journals}

Édition électronique

URL : http://journals.openedition.org/civilisations/355

DOI : $10.4000 /$ civilisations. 355

ISSN : 2032-0442

Éditeur

Institut de sociologie de l'Université Libre de Bruxelles

Édition imprimée

Date de publication : 1 avril 2006

Pagination : 85-93

ISBN : 2-87263-006-6

ISSN : 0009-8140

Référence électronique

Léon de Saint Moulin, « 35 ans de recherches en RDC », Civilisations [En ligne], 54 | 2006, mis en ligne

le 01 avril 2009, consulté le 03 mai 2019. URL : http://journals.openedition.org/civilisations/355 ; DOI :

10.4000/civilisations.355

(c) Tous droits réservés 


\title{
35 ans de recherches en $R D C$ \\ Permanences et renouvellement des méthodes
}

\author{
Léon de SAINT MOULIN
}

Résumé : L'article rappelle d'abord des règles générales, qui sont souvent trop peu assimilées. La première est le sens des objectifs, qui impose une préparation approfondie des recherches : on ne peut chercher ce qu'un milieu ne peut donner, on doit interroger les gens dans les catégories de leur culture, on ne peut programmer des recherches administratives dans des schémas étrangers au fonctionnement des administrations à visiter. La connaissance du milieu et des problèmes à éclairer par les enquêtes doit donc être assurée avant la détermination de la population cible, le recrutement des enquêteurs et l'élaboration du questionnaire. De très bons résultats ont été obtenus en procédant d'abord par questions ouvertes auprès d'un nombre limité de personnes et ensuite par questionnaire auprès d'un échantillon de la population d'un périmètre déterminé. L'article explicite les règles données pour le choix des personnes interrogées tant dans le cas d'interviews libres que dans celui d'enquêtes par échantillon. Il souligne le caractère décisif de la relation de confiance à établir avec les personnes à interroger et précise certaines règles protocolaires en RDC. L'article présente ensuite, parmi les changements intervenus dans la méthodologie depuis 1990, l'apport des ordinateurs.

Mots-clés : culture, interview libre, échantillon, organisation administrative, questionnaire.

Summary: This article starts by reviewing general research rules that are often underestimated. The first pertains to the very meaning of the research itself. This entails serious background preparation because it is senseless to investigate beyond what an environment can give; because people have to be interviewed in categories that relate to their culture and because programming administrative research is meaningless in patterns that are alien to the administrative systems under study. Knowledge of the research area and subject and problems that need to be clarified has to be insured prior to identifying a target group, recruiting researchers or elaborating a questionnaire. Very solid results have been obtained by proceeding first with open questions amongst a small group and subsequently by administering questionnaires to a circumscribed sample of individuals. The article specifies rules for the choice of research subjects, either in open interviews or in sample studies. It emphasises the importance of establishing relations of trust with people being interviewed and pinpoints certain Congolese formalities. In a final section, major evolutions since 1990, such as the advent of the computer, are discussed.

Key words: culture, open interview, sample, administrative organisation, questionnaire. 
$\mathrm{E}$ n résidence au Congo depuis 1967, j'y ai d'abord travaillé trois ans en tant que chargé de recherches du FNRS, avec un mandat d'étude sur l'évolution des villes. J'étais docteur en histoire, avec une thèse d'histoire sociale qui m'avait imposé un apprentissage des sciences sociales. Mais j'ai beaucoup appris en ce domaine et j'ai acquis une première autorité pour avoir été associé à l'enquête socio-démographique de Kinshasa organisée par la Coopération française avec l'Institut national de la statistique et pour avoir ensuite réalisé avec l'Institut géographique du Congo la mise à jour du plan de la ville de Kinshasa. Depuis ces jours, je sais qu'il existe au Congo un personnel capable de beaucoup de courage et de savoir-faire dans les travaux de terrain, ainsi que des dirigeants capables de les apprécier et avec lesquels un dialogue franc est assez facile.

De 1970 au milieu des années 1980, j'ai assumé des cours et des fonctions de responsabilité à l'université, dont huit ans à Lubumbashi. J'ai dirigé des travaux d'étudiants à la Faculté des lettres et en sciences sociales, sur l'histoire de la population et de l'organisation administrative, principalement. Mais c'est surtout depuis mon entrée en service aux Facultés catholiques de Kinshasa en 1985 que j'ai développé des cours et séminaires d'analyse sociale dans le cadre desquels j'ai organisé une trentaine d'enquêtes urbaines, parfois socio-démographiques, mais le plus souvent d'opinion (de Saint Moulin 1995, 1996, 1997, 1998, 2000, 2002).

En 2002, la Fondation Konrad Adenauer m'a demandé de réaliser à l'échelle du pays une enquête sur la perception de la démocratie et de l'Etat de droit (de Saint Moulin et al. 2003). Je l'ai fait de façon double, avec l'appui des autorités de l'Université de Kinshasa. D'une part, j'ai envoyé des assistants de la Faculté des sciences sociales et politiques pour une recherche par questions ouvertes dans les provinces du Bas-Congo, du NordKivu et du Kasai Oriental. D'autre part, j'ai moi-même entrepris avec des étudiants de licence de la même Faculté une enquête par sondage sur l'ensemble de la ville de Kinshasa, par questions fermées. En 2005, sort de presse un Atlas de l'organisation administrative de la $R D C$, qui donne pour chaque territoire (il y en a 145), sa subdivision en collectivités (il y en a 737) et en groupements (il y en a 5 397) (de Saint Moulin 2005). C'est l'aboutissement de nombreuses recherches de bibliothèque, mais aussi d'archives et de terrain. Les réflexions méthodologiques que m'inspire cette expérience sont les suivantes.

\section{Permanence des règles fondamentales : le sens des objectifs}

D’une part, on ne méditera jamais assez les règles fondamentales de la recherche : il faut bien définir ce qu'on cherche et se demander si la méthodologie qu'on veut appliquer peut fournir les informations dont on a besoin. J'ai moi-même dû progresser dans la compréhension de ces règles et j'ai été témoin de trop d'enquêtes lancées sans préparation suffisante et avec une méthodologie inadéquate pour croire superflu de les rappeler.

La préparation d'une enquête suppose une maîtrise suffisante des notions en jeu dans le problème à étudier et une définition adéquate des personnes à interroger. Des enquêtes sur la parenté ou les ménages ne peuvent être entreprises dans des cadres qui ne correspondent pas à la culture de ceux qu'on va interroger. Si on veut utiliser le facteur religieux dans son analyse, on ne peut se contenter des déclarations d'appartenance sans 
étude préalable des groupes existant dans le milieu à étudier. Des recherches de données administratives ne peuvent être programmées selon des schémas étrangers au mode de fonctionnement et aux catégories de travail des administrations à visiter. Le chercheur doit apprendre à chercher et apprécier ce qu'il peut trouver et non imposer à d'autres la charge de produire les nouvelles données dont il a besoin, notamment pour des comparaisons internationales.

Les questions posées doivent par ailleurs éclairer tous les aspects de la réalité à étudier. On oublie souvent de bien spécifier les informations de fait dont on a besoin, notamment concernant l'identité, l'environnement et l'histoire de celui qu'on interroge. Même dans des enquêtes d'opinion, des données de fait sont essentielles. Et de même dans une enquête socio-économique, des questions culturelles - de perception et d'appréciation - sont aussi nécessaires. S'il faut éviter de se disperser, il faut ouvrir suffisamment la gamme des questions posées pour éclairer aussi les problèmes connexes à celui qui fait l'objet direct de la recherche.

Si la règle de base est d'enregistrer aussi fidèlement que possible les déclarations de la personne interrogée, cela ne dispense pas d'un regard critique. Dans les questions de fait, principalement, on doit pouvoir établir de façon critique ce qu'on affirmera au terme des enquêtes. Le caractère plus ou moins sincère et spontané des réponses doit aussi pouvoir être établi. De ce point de vue, les enquêtes auprès de personnes simples sont beaucoup plus aisées qu'auprès des plus instruites, qui donnent souvent des réponses apprises et se préoccupent particulièrement de donner une «bonne réponse ». Pour satisfaire à ces exigences, des indications méthodologiques détaillées doivent être demandées aux enquêteurs (rapports d'enquête) et le maximum de précisions doit être fourni dans la présentation des résultats sur la forme des questions posées (formulation et mode d'interview) et les réponses qu'elles ont suscitées.

\section{Questions ouvertes et questions fermées}

Il est stérile d'opposer en principe les deux grands types d'enquêtes d'opinion, par questions ouvertes et par questions fermées. Chacun a son utilité et l'expérience m'a montré l'intérêt de combiner les deux. Le premier type a un caractère exploratoire très efficace et est une excellente préparation pour le second. Les questions ouvertes doivent être très générales, pour laisser la personne interrogée s'exprimer dans ses propres catégories. Il ne faut poser des sous-questions que dans la mesure où l'interviewé s'écarte du sujet de l'enquête ou omet de s'exprimer sur des aspects importants de la question. Il faut au contraire laisser celui qui parle exprimer ce qui lui tient à cœur, pour éviter de ne retrouver à la fin de l'interview que ses propres schémas de pensée.

Les enquêteurs doivent avoir une bonne formation générale, mais leur formation spécifique peut se faire très rapidement : ils doivent comprendre le but poursuivi (faire l'inventaire de toutes les opinions en circulation dans un milieu donné), la règle essentielle (de diversifier au maximum les personnes interrogées) et la façon de noter les réponses obtenues (en reprenant autant que possible les expressions employées, les exemples et les symboles proposés, et les éléments émotifs qui peuvent avoir accompagné le discours). J'ai souvent demandé à chaque étudiant cinq à sept interviews pour lesquelles il devait remettre un rapport distinct, avec les éléments requis concernant le mode de rencontre de la personne et son identité, c'est-à-dire ce qui permettra de comprendre qui dit quoi. J'ai aussi souvent demandé qu'ils réunissent une dizaine de personnes pour les interroger en groupe, en laissant chacun réagir aux déclarations des autres (panel). Ce type d'enquêtes 
est d'autant plus fécond que les personnes réunies sont d'identités sociales variées. Le rapport de ces panels doit suivre le déroulement du débat, mais il doit aussi identifier les intervenants et donner finalement une vision assez personnalisée de chacun. La seule base de généralisation des informations recueillies par ce type d'enquête est en effet la logique des réponses : pourquoi telle personne dit-elle ceci ou cela?

De telles enquêtes sont malheureusement longues à dépouiller. C'est un travail d'analyse de contenu, où il faut identifier des unités minimales de sens et s'efforcer de comprendre ensuite leur articulation. A la question qu'est-ce que la démocratie? on peut ainsi distinguer les personnes qui s'expriment surtout en termes de liberté et de participation, mais en RDC, on trouvera aussi celles qui insistent sur la dimension sociale: un système politique qui fonctionne pour le bien-être de la majorité. Dans ces registres, on entendra beaucoup d'expressions en langues africaines. Au niveau de l'articulation des notions de base, on entendra davantage de réponses apprises, telles que la formule de Lincoln et un discours sur l'Etat de droit, mais on doit s'efforcer de comprendre les priorités de chacun en fonction de son expérience vécue et de son système de valeurs. A l'est du Congo, l'aspiration la plus profonde en 2002 était la paix. Dans des centres commerçants pleins d'initiative, comme Butembo et Mbuji-Mayi, la liberté était davantage valorisée que dans d'autres milieux. Au Bas-Congo, où un mouvement de valorisation culturelle autonome était en cours de répression, l'expression « démocratie inculturée » a été forgée pour traduire l'aspiration à un système politique qui fasse plus de place aux préoccupations de la population locale.

Ces enquêtes par questions ouvertes ont une grave limite : comme elles sont longues à dépouiller, on ne les fait jamais qu'auprès d'un nombre assez limité de personnes. Elles n'atteignent donc jamais un échantillon représentatif d'une population et ne permettent pas de mesurer le poids respectif des différentes opinions enregistrées. C'est une tentation à laquelle il faut résister de résumer les résultats de telles enquêtes en termes d'opinion « moyenne » ou « dominante ». Si on veut pouvoir établir le poids de diverses composantes dans une opinion, par exemple la place des préoccupations économiques et morales dans la perception du mal, il faut donc procéder à un autre type d'enquête, permettant d'atteindre un nombre suffisant de personnes pour qu'elles constituent un véritable échantillon de la population cible. On recourt à un questionnaire à choix multiple, dont le dépouillement est devenu relativement facile par ordinateur. L'inconvénient, souvent souligné, de contraindre les interviewés à s'exprimer dans des catégories qui ne sont pas les leurs, est fortement réduit si les réponses proposées sont issues d'une enquête préliminaire par questions ouvertes.

La préparation d'un questionnaire d'enquête appelle de toutes façons une attention plus grande qu'on ne le croit habituellement. Une pré-enquête auprès d'un premier groupe de personnes peut servir de test. Elle est particulièrement recommandable à ceux qui ne sont pas familiers du milieu à étudier, mais elle peut éviter à tous des formulations maladroites, qui peuvent être mal comprises ou embarrasser. Pour apprécier l'intérêt des questions et leur couverture des divers aspects du problème à étudier, il est bon de se demander quelle conclusion pourrait découler de tel ou tel choix des items proposés et quels peuvent en être les facteurs d'explication, sur lesquels il importerait donc de recueillir des informations.

La préparation d'une enquête par questionnaire comporte un autre volet dont les déficiences peuvent aussi réduire fortement la qualité des résultats, c'est l'établissement de l'échantillon à interroger. Chacun sait qu'on ne peut parler d'échantillon que si tous les 
membres de la population cible ont eu les mêmes chances de figurer parmi les personnes interrogées. On y arrive par un tirage aléatoire ou systématique (s'il n'y a pas de récurrence du même ordre que la raison de la progression adoptée) sur une liste de base.

L'enquête socio-démographique au dixième de Kinshasa en 1967 avait effectivement atteint des nombres correspondant au dixième des effectifs connus des salariés des grandes entreprises de la ville et des élèves des différentes classes du primaire et du secondaire. Cette enquête avait été construite sur base d'une liste indirecte de la population, c'està-dire d'un relevé parcellaire exhaustif. Le tirage des parcelles dont tous les habitants avaient ensuite été recensés a été fait après numérotation continue des parcelles dans chaque portion socio-administrative du territoire pour lequel on voulait établir des chiffres et des caractéristiques de la population. Dans les buildings, l'appartement avait été considéré comme l'unité parcellaire, mais il y en avait très peu. Un problème plus délicat a été celui des populations dites comptées à part, c'est-à-dire celles des habitats collectifs tels que les camps militaires, les homes d'étudiants et les centres de détention. Leurs caractéristiques n'ont pas été établies, mais leur nombre a été recueilli auprès des autorités compétentes et ajouté aux résultats des enquêtes. L'échantillon de la plupart des enquêtes que j'ai réalisées par sondage a eu comme première base un échantillonnage des parcelles du ou des quartiers cibles.

Dans des quartiers bien circonscrits, le travail d'inventaire des parcelles habitées n'est pas trop lourd à réaliser, mais il ne peut être improvisé pour une grande étendue telle qu'une ville. Pour mes enquêtes à Kinshasa, le plan réalisée en 1969 reste valable pour les parties anciennes de la ville et je dispose de la couverture aérienne de 1985 annotée, ainsi que d'une image satellitaire de 1995 bien étudiée pour les quartiers périphériques. C'est sur ces bases que j'attribue aux étudiants la ou les rues dans lesquelles ils doivent enquêter pour les recherches que j'organise avec eux. Comme on avait souligné l'importance de la connaissance du milieu pour une bonne rédaction des questions, il faut affirmer celle de la connaissance du terrain pour la constitution de la base d'un sondage. Cela peut s'acquérir ou être construit par un étranger, mais c'est un travail préparatoire qu'on ne peut minimiser.

Pour des enquêtes d'opinion, seules les personnes adultes sont à interroger. Je prends en général comme norme d'avoir dix-sept ans depuis le 31 décembre de l'année précédant l'enquête et j'applique la règle habituelle des quotas par sexe et par âge, du dernier recensement disponible (du 30 juin 1984). Les enquêteurs reçoivent ainsi comme double règle de choix des personnes à interroger celle de les choisir à raison d'une seule personne par parcelle (pour éviter les effets de grappe) en déterminant le choix des parcelles selon une progression qui les fait répartir de façon égale sur toute la ou les rue(s) qui leur sont attribuées, et dans les parcelles retenues de procéder par quotas. En pratique, cela permet à l'enquêteur d'interroger dans la première parcelle visitée n'importe quelle personne âgée d'au moins dix-sept ans, mais le choix se resserre ensuite au fur et à mesure de l'épuisement des quotas imposés pour les divers groupes. Cela facilite le début du travail tout en contraignant l'enquêteur à ne pas se laisser influencer par les facilités ou les difficultés de contact.

Je retiens habituellement quatre groupes d'âges : les 17-24, 25-34, 35-44 et 45 ans et plus. Les enquêteurs devant interroger un nombre entier de personnes de chaque catégorie, de légères distorsions de l'échantillon peuvent résulter des quotas imposés, surtout si le nombre d'enquêtes effectuées par chacun est inférieur à vingt. Les étudiants réussissent 
cependant à appliquer des directives du genre trois ou quatre hommes de 25 à 34 ans et deux ou trois hommes de 35 à 44 ans : cela donne une moyenne proche de 3,5 et 2,5 hommes de chaque catégorie.

L'écueil principal rencontré dans l'expérience est celui des biais que peut introduire quelqu'un qui est pris comme accompagnateur par un enquêteur étranger au milieu. Sans que les mécanismes aient toujours pu en être établis, ce procédé a plusieurs fois conduit à des distorsions évidentes dans la proportion des personnes de diverses catégories interrogées. Il y a un risque général de sur-représentation parmi les personnes interrogées des catégories sociales les plus proches de l'enquêteur ou de son guide et de sous-représentation des personnes qui en diffèrent davantage ou qui s'expriment plus difficilement (par défaut d'instruction ou handicap). De ce point de vue, la qualification des enquêteurs est importante : j'estime avoir obtenu la meilleure image de la population de Kinshasa dans l'enquête réalisée en 2002 par des étudiants de licence en sciences sociales fortement encadrés par des assistants.

Pour une raison semblable, s'il est relativement aisé de constituer un échantillon de l'ensemble des membres d'une population, par les méthodes indiquées, il est très difficile de prétendre y avoir réussi si on limite son enquête à une catégorie déterminée, par exemple aux adhérents d'une religion déterminée ou aux diplômés universitaires.

\section{Importance d'une relation de confiance}

Last but not least. Parmi les règles qu'on peut trouver dans tous les manuels mais qu'il me semble utile de rappeler, je mentionnerai enfin l'importance d'établir une relation de confiance avec la personne à interroger. Le bon enquêteur est pour une part celui qui est efficace et peut réaliser une dizaine d'enquêtes par jour. Mais il doit aussi susciter la confiance et ramener des réponses sincères ... et ne pas susciter des oppositions qui peuvent appeler des démarches réparatrices parfois laborieuses. On y arrive en se présentant poliment, en expliquant l'objet de sa visite et en s'intéressant discrètement à la personne visitée. Il est essentiel de voir si le moment est propice à l'enquête. Par ailleurs, comme ce qui est recherché est l'opinion spontanée de la personne, il est contreindiqué de lui laisser remplir le questionnaire comme une feuille d'examen et de venir le reprendre le lendemain.

En RDC, une visite d'information aux autorités est en général utile et parfois nécessaire. La mentalité de la population a de nombreux traits du genre homo hierarchicus et un enquêteur qui n'a pas l'aval des instances régulatrices concernées suscite la méfiance (Dumont 1979). Une visite de courtoisie au chef de quartier ou de village est donc à recommander et éventuellement au bourgmestre ou même au Gouverneur. Les enquêteurs sont eux-mêmes désireux d'avoir un document établissant le caractère académique ou officiel de leur démarche.

\section{Les changements intervenus dans la méthodologie depuis 1990}

Les ordinateurs ont apporté un changement radical dans la préparation et le dépouillement des enquêtes. Il est désormais facile et rapide de codifier des réponses choisies sur une grille à choix multiples. On peut dès lors multiplier les dépouillements par catégories (hommes/femmes, jeunes/plus âgés, célibataires/autres, diplômés d'état/ autres, étudiants/ayant un emploi/sans emplois, catholiques/autres, etc.). Cela oriente l'analyse sur les facteurs de variation des opinions. 
Il y a des exigences techniques, c'est-à-dire au minimum le calcul des différences significatives entre les réponses des diverses catégories, parfois le calcul de corrélations, et éventuellement une analyse factorielle. Mais celles-ci appellent les informations appropriées. On doit donc systématiser plus que jadis les éléments d'identité des personnes interrogées en fonction des analyses qu'on désire ensuite pouvoir mener. On retrouve sous une nouvelle forme la règle fondamentale d'une claire vision des objectifs d'une enquête. Dans la codification des enquêtes, j'ai en général autant de chiffres pour les éléments d'identité que pour la codification des réponses.

Ce travail d'ordinateur est sensiblement accéléré ou ralenti par une plus ou moins bonne disposition des informations enregistrées et leur codification. En général, tous mes questionnaires tiennent sur une feuille A4 recto verso, avec un encadré au début pour les éléments d'identité et un autre à la fin pour des observations et indications complémentaires éventuelles. Les indications complémentaires sont en général peu nombreuses, mais elles sont parfois précieuses : elles peuvent aider à interpréter certaines réponses. Elles sont cependant rarement codifiables comme telles et appellent donc une analyse spécifique.

Un relèvement sensible du niveau général d'instruction de la population et la dégradation de ses conditions de vie sont d'autres changements intervenus dans les travaux d'enquête depuis 1990. La dégradation peut concerner très directement les recherches. Des dossiers qui existaient jadis ont disparu ou ne sont plus tenus à jour. La population, par ailleurs, qui répondait jadis fort spontanément et avec de grandes espérances aux questions des enquêteurs, est parfois aujourd'hui méfiante et construit plus souvent ses réponses avec des arrière-pensées. Au niveau des enquêteurs, par contre, l'évolution est tout bénéfice. Il est plus facile que jadis de recruter des collaborateurs qualifiés pour des enquêtes et ils acceptent tout autant que jadis la sélection sur base des réalisations et le contrôle par des superviseurs.

Mais le changement le plus significatif est sans doute la revalorisation du culturel dans les analyses sociales. Jean Paul II (1991), qui a largement repris l'analyse marxiste dans son discours social, observe avec finesse :

\section{Il n'est pas possible de comprendre l'homme en partant exclusivement du domaine de l'économie ... On comprend l'homme d'une manière plus complète si on le replace dans son milieu culturel, en considérant sa langue, son histoire, les positions qu'il adopte devant les événements fondamentaux de l'existence, comme la naissance, l'amour, le travail, la mort ... Au fond, les cultures des diverses nations sont autant de manières d'aborder la question du sens de l'existence personnelle.}

Pierre Bourdieu, par la sociologie de l'éducation notamment, a aussi revalorisé le culturel dans l'analyse sociale. Et pourtant, j'ai cru devoir conclure des enquêtes sur $L a$ perception du salut et de la libération à Kinshasa en opposant les conclusions auxquelles elles me conduisaient à celles de ses célèbres recherches sur La misère du monde (de Saint Moulin 1997). Il est certain que la population de Kinshasa a une spiritualité plus riche que celle de la France, mais il me semble que l'approche adoptée était aussi responsable de ce que je considère comme une pauvreté dans les réponses enregistrées. Même dans une enquête par questions ouvertes, mais a fortiori dans une démarche par questions fermées, le risque ne peut être minimisé d'enfermer les personnes interrogées dans le cadre de ses propres catégories. Ainsi revient une fois de plus la règle fondamentale du sens des objectifs : une enquête n'a son plein sens que si elle cherche à comprendre aussi profondément que possible la population qu'elle interroge; elle est biaisée si elle est construite pour démontrer la valeur des opinions de celui qui l'organise ou, tout 
simplement, si elle est dominée par ces opinions. Cela signifie qu'on ne peut en rester à un idéal d'objectivité scientifique d'ordre rationnel. La réalité a aussi une subjectivité dont il importe de rendre compte avec fidélité, ce qui suppose respect et même sympathie.

En un sens, les recherches au Congo, comme partout ailleurs, seront d'autant plus riches qu'elles auront été abordées avec un regard bienveillant, prêt à découvrir des logiques, des ressources émotives et un mode de vie assez différent des siens. De ce point de vue, malgré les satellites et l'ampleur de la documentation qui peut être accumulée à l'étranger, il ne fait pas de doute que les informations les plus riches concernant le Congo se trouvent au Congo. Puisse donc le travail de terrain s'y développer avec des méthodes de plus en plus efficaces.

\section{Les résultats des enquêtes}

Chacun sait qu'après la préparation et la réalisation d'une enquête, il reste à en produire les résultats sous une forme utile. Les personnes interrogées demandent souvent à pouvoir accéder aux résultats de la recherche et un large intérêt est suscité chaque fois qu'une conférence est organisée à ce propos dans le milieu enquêté. Les étudiants ayant réalisé une enquête se sentent aussi valorisés si les résultats en sont publiés. Je l'ai délibérément fait chaque fois que c'était possible dans des revues ou des collections publiées au Congo, principalement Congo-Afrique du CEPAS, la Revue africaine de théologie et les Cahiers des religions africaines des Facultés catholiques de Kinshasa.

La télévision a une large audience à Kinshasa et cette audience s'est étendue à toutes les villes du pays dans les années 1980 et au début des années 1990. Le fait d'avoir pu y présenter plus de cinquante émissions, largement alimentées par les travaux de terrain et pour une part directement consacrées à des résultats d'enquêtes, m'a donné une image familière pour beaucoup de Congolais. Ce qui est perçu dans les émissions n'est cependant pas nécessairement ce sur quoi on a insisté. Il est même certain que l'implicite a plus de poids dans la perception sociale que l'explicite et que les mécanismes d'appropriation permettent à la population de lire un message à contre-sens de l'intention de son émetteur. Ce qui marque le plus la perception est le fait que vous, avec votre identité, êtes apparu dans les médias avec tel genre de discours sur tel genre de problème.

Il ne fait pas de doute que beaucoup de résultats d'enquêtes que j'ai diffusés ont été appropriés par la population kinoise, et dans une certaine mesure par la population congolaise en général, et font aujourd'hui partie de sa culture. Il y a parfois eu des essais de manipulation de données tirées de leur contexte, mais, dans le genre de travaux que j'ai réalisés, je loue la maturité de la population congolaise qui a en général fort bien perçu la signification des données présentées. 


\section{Références bibliographiques}

DE Saint Moulin, Léon

1995. «La perception du mal à Kinshasa et dans quelques localités du Zaïre », Revue africaine de théologie, 19 (37), pp. 53-92.

1996. «Kinshasa trente ans après. Une enquête sur la perception sociale de la justice », Zaïre-Afrique, 36 (305), pp. 197-220.

1997. «La perception du salut et de la libération à Kinshasa », Revue africaine de théologie, 21 (425), pp. 203-233.

1998. « La mondialisation dans la commune de Ngaba à Kinshasa », Congo-Afrique, 38 (327), pp. 419442.

2000. « Eglise et société dans l'opinion publique à Kinshasa/Kalamu », Revue africaine de théologie, 24 (47-48), pp. 117-148.

2002. «La place de la religion dans la vie selon une enquête effectuée à Kinshasa/Lemba Sud » CongoAfrique, 367, pp. 415-433.

De SAint Moulin, Léon, en collaboration avec Ch. Kabongo Mponga, 2000. « La perception des identités et des responsabilités sociales à Kinshasa. Résultats d'une enquête au sud des communes de Lemba et de Kisenso », Congo-Afrique, 40 (346), pp. 355-379.

DE SAINT Moulin, Léon, en collaboration avec Jean-Luc Kalombo, 2005. Atlas de l'organisation administrative de la RDC. Kinshasa.

DE Saint Moulin, Léon, en collaboration avec J.M. Kinkela, N. Paluku et E. Tshimanga, 2003. La perception de la démocratie et de l'Etat de droit en RDC. Kinshasa : CEPAS.

Dumont, Louis, 1979. Homo hierarchicus; le système des castes et ses implications. Paris : Gallimard. En opposition à Homo aequalis, du même auteur. Paris : Gallimard, 1977.

Jean Paul II, 1991. Centesimus annus, lettre encyclique à l'occasion du centenaire de Rerum novarum, 24. 
